\title{
Time to Failure on Oral Glucose-Lowering Agents for Patients with Type 2 Diabetes: A Retrospective Cohort Study
}

\author{
Kristina S. Boye · Maureen J. Lage (D) · Jacek Kiljański
}

Received: January 5, 2021 / Accepted: March 17, 2021 / Published online: April 10, 2021

(C) The Author(s) 2021

\begin{abstract}
Introduction: Research has shown that glycemic control is associated with lower rates of microvascular and long-term cardiovascular complications. In the analyses reported here, we examined treatment failure on oral glucoselowering agents (GLAs), defined as having sustained hemoglobin A1c (HbA1c) $\geq 7 \%$.

Methods: This study utilized the IBM ${ }^{\circledR}$ MarketScan ${ }^{\circledR}$ Claims and Laboratory Data from 1 January 2012 through 30 June 2018. Adults with type 2 diabetes (T2D) were classified based upon the maximum number of classes of GLAs prescribed per day during the time period from 1 July 2012 through 31 December 2012. Patients were followed for 5.5 years in order to examine
\end{abstract}

Supplementary Information The online version contains supplementary material available at https:// doi.org/10.1007/s13300-021-01051-9.

K. S. Boye

Eli Lilly and Company, 893 Delaware Street, Indianapolis, IN 46225, USA

e-mail: boye_kristina_secnik@lilly.com

M. J. Lage $(\bowtie)$

HealthMetrics Outcomes Research, 27576 River

Reach Drive, Bonita Springs, FL 34134, USA

e-mail: lagemj@hlthmetrics.com

J. Kiljański

Eli Lilly Polska Sp. z o.o (Eli Lilly and Company), u.l.

Żwirki I Wigury 18a, 02-092 Warsaw, Poland

e-mail: kiljanski_jacek@lilly.com time to failure on oral GLAs, defined based upon receipt of $\geq 2$ consecutive $\mathrm{HbA1c}$ results $\geq$ $7 \%$. Multivariable analyses employing a Cox proportional hazards model were used to examine time to failure overall and based upon the number of index classes of oral GLAs prescribed. For patients who had sustained HbA1c above the threshold, multivariable analyses examined the duration of time that HbA1c remained above the threshold (i.e, glycemic burden) and whether or not an additional oral or injectable class of GLA was added to the patient treatment regimen (i.e., clinical inertia). Results: A total of 4156 patients were included in the study, of whom $16 \%$ were identified with sustained $\mathrm{HbA} 1 \mathrm{c} \geq 7 \%$ after 365 days (1 year) and $36 \%$ after 730 days ( 2 years), with half of all patients having sustained HbA1c above target after 1102 days (3 years). There was a statistically significant difference in time to having sustained HbA1c above target based upon index classes of therapy, with patients treated with more GLAs being quicker to have HbA1c above target $(P<0.0001)$. Among those patients who were found to have sustained $\mathrm{HbA} 1 \mathrm{c} \geq 7 \%$, the average number of days in the post-period that HbA1c remained above target was 1026 (2.8 years). Only $36 \%$ of patients with sustained HbA1c above target added a GLA to their treatment regimen and, for patients who did add such a therapy, the average duration from identification of HbA1c above target until treatment intensification was 401 days 
(1.1 years). Multivariable analyses revealed that, among those with sustained $\mathrm{HbA} 1 \mathrm{c} \geq 7 \%$, treatment with more classes of oral GLAs was associated with a significantly higher glycemic burden and significantly lower odds of clinical inertia.

Conclusion: These results indicate that for many patients treated with oral GLAs, glycemic control is not consistently achieved. For patients with above-target $\mathrm{HbA1c}$, the results indicate a relatively large glycemic burden and clinical inertia towards treatment intensification. The findings illustrate some limitations associated with treatment of $\mathrm{T} 2 \mathrm{D}$ with oral GLAs.

Keywords: Clinical inertia; Glucose-loweringagents; Glycemic burden; HbA1c; Kaplan-Meier analyses; Oral therapy; Type 2 diabetes

\section{Key Summary Points}

\section{Why carry out this study?}

Despite the advantages associated with glycemic control, previous research has found that treatment delays are common in the treatment of type 2 diabetes (T2D).

This study examines the relationship between treatment with oral glucoselowering agents (GLAs) and glycemic control.

\section{What was learned from the study?}

Among the 4156 individuals included in the study, half of all patients were found to have sustained $\mathrm{HbA1c}$ above the target level, defined as $\geq 2$ sequential hemoglobin A1c ( HbA1c) results above target, after 3 years; and among such patients, the average number of days that HbA1c remained above target was 1026 (2.8 years).
Only $36 \%$ of patients with sustained HbA1c above target added a GLA to their treatment regimen, and for patients who did add such a therapy, the average number of days from identification of HbA1c above target until such treatment intensification was 401 days (1.1 years).

Multivariable analyses revealed that, among those with sustained $\mathrm{HbA1c} \geq 7 \%$, treatment with more classes of oral GLAs was associated with a significantly higher glycemic burden and significantly lower odds of clinical inertia.

The results of this study illustrate the large glycemic burden and clinical inertia towards treatment intensification associated with the use of oral GLAs.

\section{DIGITAL FEATURES}

This article is published with digital features, including a summary slide, to facilitate understanding of the article. To view digital features for this article go to https://doi.org/10.6084/ m9.figshare.14223017.

\section{INTRODUCTION}

Type 2 diabetes (T2D) is a progressive disease characterized by deteriorating glycemic control due to declining beta-cell function and increasing insulin resistance [1]. Oral glucoselowering agents (GLAs) are the first-line of treatment for $\mathrm{T} 2 \mathrm{D}$, yet many patients require additional therapies to achieve glycemic control as T2D advances [2]. For example, the UK Prospective Diabetes Study (UKPDS) found that, after 6 years of treatment on oral GLAs, $44 \%$ of patients required additional therapy to manage their hyperglycemic symptoms or bring their fasting plasma glucose to $<15.0 \mathrm{mmol}$ per liter [3]. Similarly, the Diabetes Outcome Progression Trial (ADOPT) reported substantial 5-year failure rates for specific oral agents, including 
rosiglitazone (15\%), metformin (21\%), and glyburide (34\%), with failure was defined as fasting plasma glucose $>10.0 \mathrm{mmol}$ per liter [4].

While various measures of glycemic control are used [1], the American Diabetes Association (ADA) has deemed hemoglobin A1c (HbA1c) the primary such measure and has set a standard treatment goal of $\mathrm{HbA} 1 \mathrm{c}<7 \%$ for most nonpregnant adults [5]. The benefits of achieving $\mathrm{HbA} 1 \mathrm{c}<7 \%$ in patients with $\mathrm{T} 2 \mathrm{D}$ include lower rates of both microvascular and long-term cardiovascular complications [6, 7], and setting even tighter HbA1c goals might also be associated with improved microvascular outcomes [8]. Nevertheless, more intensive glycemic control has been associated with increased rates of hypoglycemia [8, 9], and fear of hypoglycemia and other concerns have been associated with a reluctance to start or intensify antidiabetic treatment in a timely way [10]. More than 50 studies [11] published over the past 2 decades have shown that such treatment delays, deemed clinical inertia [12], are common in the treatment of T2D. However, little research has solely examined treatment failure on oral GLAs, as a medication class, or subsequent outcomes.

Given the evidence of the progressive nature of diabetes, the limitations of oral GLA therapy, and clinical inertia in the treatment of T2D, we hypothesized that oral antidiabetic therapies often fail to sustain the target HbA1c over time among US patients with T2D, and that treatment intensification after such failure is often delayed. To test these hypotheses, the present retrospective analyses focused on a population of patients with T2D who were initiated on oral GLAs to determine: (1) the time to treatment failure on oral therapy, defined as the mean number of days from prescription of oral GLA medications to the record of an $\mathrm{HbA} 1 \mathrm{c} \geq 7 \%$; (2) the glycemic burden, defined as the mean number of days that patients sustained HbA1c $\geq 7 \%$; and (3) clinical inertia, defined as the mean number of days from identification of above-target HbA1c to modification of treatment.

\section{METHODS}

The study data was from the IBM ${ }^{\circledR}$ MarketScan ${ }^{\circledR}$ Claims and Laboratory Data (IBM Watson Health, Cambridge, MA, USA). The claims data consisted of Commercial Claims and Encounters (CCAE) and Medicare Supplemental (MDCR) databases. These databases provided information on patient demographic characteristics, enrollment, inpatient services, outpatient services, prescription drug use, and gross payments to a service provider. The MarketScan Laboratory database provided laboratory test results, including HbA1c test results, for a subset of patients included in the MarketScan ${ }^{\circledR}$ CCAE and MDCR databases. The data came from insurance claims and medical encounters dated from 1 January 2012 through to 30 June 2018. All study data were fully de-identified and Health Insurance Portability and Accountability Act (HIPAA) compliant. Given the use of retrospective and de-identified data, ethics committee approval was not required. Permission to use the data for this study was granted by license between IBM Watson Health and Eli Lilly and Company.

For inclusion in the study, patients were required to have filled a prescription for $\geq 1$ oral GLAs over the period from 1 July 2012 through 31 December 2012 (i.e., the identification window). For each day in the identification window, the number of classes of GLAs prescribed was examined. The index date was defined as the first date associated with the highest number of GLA classes prescribed. The pre-period was defined as the 6-month period prior to the index date; the post-period was defined as the 5.5 years after the index date; and the study period was defined as the 6-year interval spanning both the pre- and post-periods. Patients were also required to be identified as having T2D in 2012 based upon receipt of $\geq 2$ diagnoses of T2D (International Classification of Diseases, Ninth Revision, Clinical Modification [ICD-9-CM] codes 250.x0, 250.x 2 or International Classification of Diseases, Tenth Revision, Clinical Modification [ICD-10-CM] code E11xx) or one diagnosis of T2D and no more than one diagnosis of type 1 diabetes (ICD-9-CM codes 
250.x1, 250.x3 or ICD-10-CM code E10xx). Patients were excluded from the study if they used any injectable GLA in 2012, if they were diagnosed with pregnancy or gestational diabetes (ICD-9-CM code 630.xx-679.xx, V22.xx, V23.xx or ICD-10-CM code Oxxxx, Z33xx, $\mathrm{Z} 4 \mathrm{xx}$ ) at any time over the study period, if they were younger than age 18 years at the index date, or if they did not have continuous insurance coverage over the study period. Finally, patients were required to have at least one recorded $\mathrm{HbA} 1 \mathrm{c}$ laboratory test result in the preperiod and at least four such results in the postperiod. Figure 1 illustrates how each of these inclusion and exclusion criteria affected sample size.

The primary outcome of interest was time to treatment failure on oral GLA, with failure on oral antidiabetics defined as sustained HbA1c $\geq 7 \%$. The use of a $7 \%$ HbA1c threshold is consistent with ADA guidelines which state that an appropriate goal for most adults is HbA1c $<7 \%$ [5]. In this study, sustained HbA1c $\geq 7 \%$ was defined as having at least two consecutive post-period HbA1c tests with results $\geq 7 \%$, and the first such date was defined as the failure date. This definition allows for temporary fluctuations in $\mathrm{HbA1c}$ in order to conservatively estimate whether a patient is above the HbA1c target. Time to failure was examined overall as well as by the number of classes of GLAs with which patients were treated on the index date.

In addition to examining time to failure on oral GLAs, for patients who had a sustained $\mathrm{HbA} 1 \mathrm{c} \geq 7 \%$, the analyses examined glycemic burden and clinical inertia. Glycemic burden was defined as the number of days in the postperiod where HbA1c remained above target. Consistent with previous research, if an individual had an $\mathrm{HbA} 1 \mathrm{c} \geq 7 \%$, it was assumed that HbA1c stayed above target until the next measurement [13]. For patients who had a sustained $\mathrm{HbA1c} \geq 7 \%$, clinical inertia was defined as the lack of use of any additional class of GLA therapy [13]. Note that while individuals were excluded from the analyses if they used an injectable GLA in the pre-period or identification period, such use was allowed in the 5.5 year post-period. As a result, patients who added an additional class of an oral GLA or an

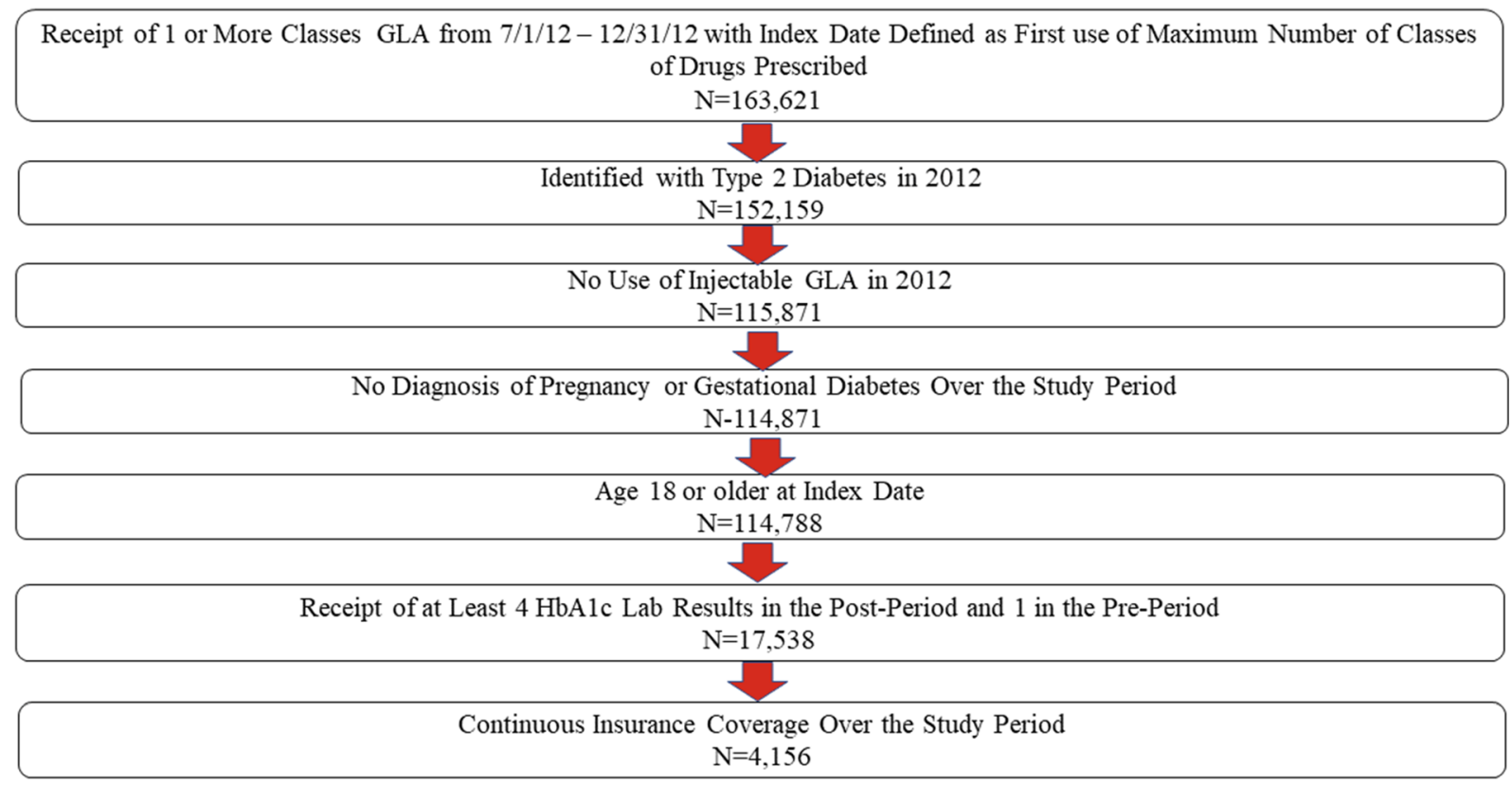

Fig. 1 Inclusion-exclusion criteria and sample size. Study period defined as the time interval from 6 months prior to the index date (pre-period) through 5.5 years post index date (post-period). GLA Glucose-lowering agent, $H b A 1 c$ hemoglobin Alc 
injectable GLA would be classified as not having clinical inertia.

For descriptive analyses, differences in categorical variables were examined using chisquare statistics, while for continuous variables differences in medians were examined using Kruskal-Wallis tests. Multivariable analyses were used to examine time to failure, glycemic burden, and clinical inertia. Specifically, time to failure was examined employing a Cox proportional hazards model. Among individuals with sustained $\mathrm{HbA} 1 \mathrm{c} \geq 7 \%$, days of glycemic burden were examined using a negative binomial model and clinical inertia was examined using logistic analyses. All multivariable analyses controlled for patient age, sex, the average number of days between HbA1c tests, and health status, as proxied by the Charlson Comorbidity Index (CCI) [14] and Diabetes Complications Severity Index (DCSI) [15]. While this research focused on the relationship between number of oral GLAs and outcomes, the complete results for the multivariable analyses are provided in Electronic Supplementary Material (ESM) Appendix A. All analyses were conducted using SAS version 9.4 (SAS Institute, Cary, NC, USA). A $P$ value $<0.05$ was considered, a priori, to be statistically significant.

\section{RESULTS}

The final sample consisted of 4156 patients. Table 1 presents the baseline characteristics for all patients and for subgroups stratified according to the number of classes of GLAs prescribed in the identification window. The median age of all patients was 55 years, and $56.0 \%$ of patients were male. Most of the individuals in this population resided in the South region of the USA (64.3\%), and patients were insured most frequently via a health maintenance organization $(43.6 \%)$ or preferred provider organization $(21.3 \%)$. In the identification window, $53.4 \%$ of patients filled a prescription for no more than one class of oral GLAs per day, 34.1\% filled a prescription for two classes of oral GLAs, and $12.4 \%$ filled a prescription for three or more classes of oral GLAs per day. As the number of index classes of oral GLAs increased, patients were more frequently male, residents of the South region of the USA, and insured through a point of service or health maintenance organization, while they were less frequently female, residents of the the North Central region of the USA, and insured with comprehensive insurance or consumer-directed health plans.The median CCI or DCSI scores were zero for all patients as well as all groups of patients based upon number of classes of oral GLAs (both $P>0.05)$.

Figure 2 presents the results of the multivariable analyses which examine the duration of time until the above-target HbA1c was identified based upon the first of $\geq 2$ HbA1c results above target. Figure $2 \mathrm{a}$ (left) shows that $16 \%$ of the overall population had a sustained HbA1c $\geq 7 \%$ after 365 days ( 1 year), $36 \%$ had a sustained $\mathrm{HbA} 1 \mathrm{c} \geq 7 \%$ after 730 days (2 years), and $50 \%$ had a sustained HbA1c $\geq 7 \%$ by 1102 days (3 years). Figure $2 \mathrm{~b}$ (right) shows that the number of classes of oral GLAs prescribed and the time to treatment failure on oral GLAs had an inverse association. For instance, among those treated with one class of oral GLAs, $50 \%$ had HbA1c $\geq 7 \%$ after day 1815 (5.0 years); among those treated with two classes of oral GLAs, 50\% had $\mathrm{HbA1c} \geq 7 \%$ after day 769 (2.1 years); and among those treated with $\geq 3$ classes of oral GLAs, $50 \%$ had HbA1c $\geq 7 \%$ after day 639 (1.8 years). As these results indicate, patients treated with more GLAs more quickly failed treatment, as measured by having a sustained HbA1c $\geq 7 \%$. Likewise, the greater the number of oral GLAs prescribed, the more likely a patient was to sustain above-target HbA1c after only 1 year. Specifically, for those treated with one, two, or three or more classes of oral GLAs, the percentage of patients with a sustained HbA1c $\geq 7 \%$ after 1 year was 11,23 , or $30 \%$, respectively, while after 2 years these percentages were 25,48 , or $58 \%$, respectively.

Figure 3 examines glycemic burden. Unadjusted descriptive statistics (Fig. 3a) show that those who sustained $\mathrm{HbA} 1 \mathrm{c} \geq 7 \%$ did so for $51.1 \%$ of the post-period (1026 days; 2.8 years) on average, with this percentage increasing as the number of classes of oral GLAs prescribed in the pre-period increased. For instance, among those prescribed one oral class of GLAs, patients 
Table 1 Descriptive statistics-overall and by number of classes of glucose-lowering agent

\begin{tabular}{|c|c|c|c|c|c|}
\hline \multirow[t]{2}{*}{ Baseline characteristics } & \multirow[t]{2}{*}{$\begin{array}{l}\text { All } \\
\text { patients }\end{array}$} & \multicolumn{4}{|c|}{$\begin{array}{l}\text { Patients characterized by number of index classes of } \\
\text { oral GLAs }\end{array}$} \\
\hline & & $\begin{array}{l}1 \text { oral } \\
\text { GLA }\end{array}$ & $\begin{array}{l}2 \text { oral } \\
\text { GLAs }\end{array}$ & $\begin{array}{l}3+\text { oral } \\
\text { GLAs }\end{array}$ & $P$ value \\
\hline Sample size & 4156 & 2221 & 1419 & 516 & \\
\hline Age, years, median $(\mathrm{IQR})^{\mathrm{b}}$ & $55(50-59)$ & $55(50-59)$ & $55(50-58)$ & $55(50-58)$ & 0.6779 \\
\hline Sex & & & & & $<0.0001$ \\
\hline Male & $\begin{array}{l}2329 \\
\quad(56.0)\end{array}$ & $\begin{array}{l}1154 \\
\quad(52.0)\end{array}$ & $841(59.3)$ & $334(64.7)$ & \\
\hline Female & $\begin{array}{l}1827 \\
(44.0)\end{array}$ & $\begin{array}{l}1067 \\
(48.0)\end{array}$ & $578(40.7)$ & $182(35.3)$ & \\
\hline Region & & & & & 0.0330 \\
\hline Northeast & $462(11.1)$ & $243(10.9)$ & $162(11.4)$ & $57(11.1)$ & \\
\hline North Central & $903(21.7)$ & $512(23.1)$ & $294(20.7)$ & $97(18.8)$ & \\
\hline South & $\begin{array}{l}2674 \\
(64.3)\end{array}$ & $\begin{array}{l}1419 \\
(63.9)\end{array}$ & $910(64.1)$ & $345(66.9)$ & \\
\hline West & $115(2.8)$ & $45(2.0)$ & $53(3.7)$ & $17(3.3)$ & \\
\hline Unknown & $2(0.1)$ & $2(0.1)$ & $0(0.0)$ & $0(0.0)$ & \\
\hline Insurance Plan Type & & & & & 0.0064 \\
\hline Comprehensive & $694(16.7)$ & $401(18.1)$ & $226(15.9)$ & $67(13.0)$ & \\
\hline Health Maintenance Organization & $\begin{array}{l}1810 \\
(43.6)\end{array}$ & $956(43.0)$ & $622(43.8)$ & $232(45.0)$ & \\
\hline Point of Service & $436(10.5)$ & $198(8.9)$ & $168(11.8)$ & $70(13.6)$ & \\
\hline Preferred Provider Organization & $884(21.3)$ & $471(21.2)$ & $299(21.1)$ & $114(22.1)$ & \\
\hline Consumer Directed Health Plan & $146(3.5)$ & $84(3.8)$ & $47(3.3)$ & $15(2.9)$ & \\
\hline Other/unknown & $186(4.5)$ & $111(5.0)$ & $57(4.0)$ & $18(3.5)$ & \\
\hline Charlson Comorbidity Index, median (IQR) ${ }^{\mathrm{b}}$ & $0(0-1)$ & $0(0-1)$ & $0(0-1)$ & $0(0-1)$ & 0.3938 \\
\hline $\begin{array}{l}\text { Diabetes Complications Severity Index, median } \\
(\mathrm{IQR})^{\mathrm{b}}\end{array}$ & $0(0-1)$ & $0(0-1)$ & $0(0-1)$ & $0(0-1)$ & 0.6087 \\
\hline
\end{tabular}

Values in table are presented as the frequency $(\mathrm{N})$ with the percentage in parenthesis, unless indicated otherwise GLA Glucose-lowering agent

a 41 patients received prescriptions for 4 classes of oral GLAs and 2 patients received prescriptions for 5 classes of oral GLAs

b For continuous variables, medians and interquartile ranges (IQR) are reported since Kolmogorov-Smirnoff test reject the hypothesis of normality 
A Time to Sustained HbA1c $\geq 7 \%$ : All Patients

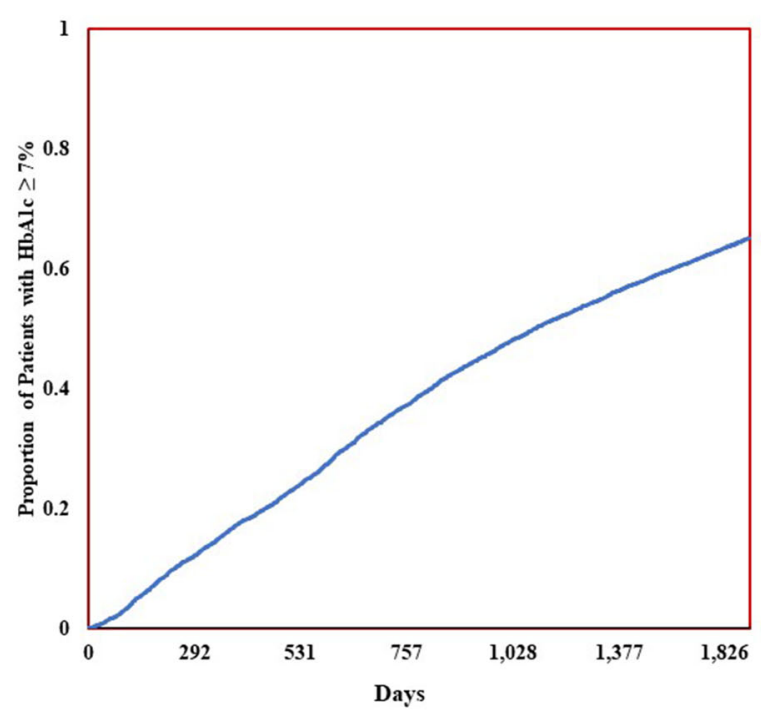

Fig. 2 Time to sustained HbAlc $\geq 7 \%$, overall and by number of classes of oral glucose-lowering agents. Time to sustained $\mathrm{HbAlc} \geq 7 \%$ was analyzed using the Cox model adjusted for age, sex, patient general health, and average

sustained $\mathrm{HbA} 1 \mathrm{c} \geq 7 \%$ for $44.8 \%$ of the 5.5 year post-period, while those prescribed $\geq 3$ oral classes sustained $\mathrm{HbA} 1 \mathrm{c} \geq 7 \%$ for $59.7 \%$ of the post-period. The multivariable analyses (Fig. 3b) are generally consistent with the descriptive statistics, with use of more classes of oral GLAs associated with a larger glycemic burden. For example, the incidence rate ratio illustrates that patients prescribed two classes of oral GLAs or three or more classes of oral GLAs, compared to those who were prescribed only one class, had 1.22-fold (incidence rate ratio [IRR] 1.216; 95\% confidence Interval [CI] 1.154-1.283) and 1.33fold (IRR 1.331; 95\% CI 1.239-1.430) more days of glycemic burden, respectively.

Figure 4 examines clinical inertia. Descriptive analyses (Fig. 4a) illustrate that, despite having a sustained HbA1c above target, $64 \%$ of the patients in this population were not prescribed any additional glucose-lowering therapies over the entire 5.5 year post-period. This percentage decreased as the number of classes of
B Time to Sustained HbA1c $\geq 7 \%$ :

By Number of Classes of Oral GLAs

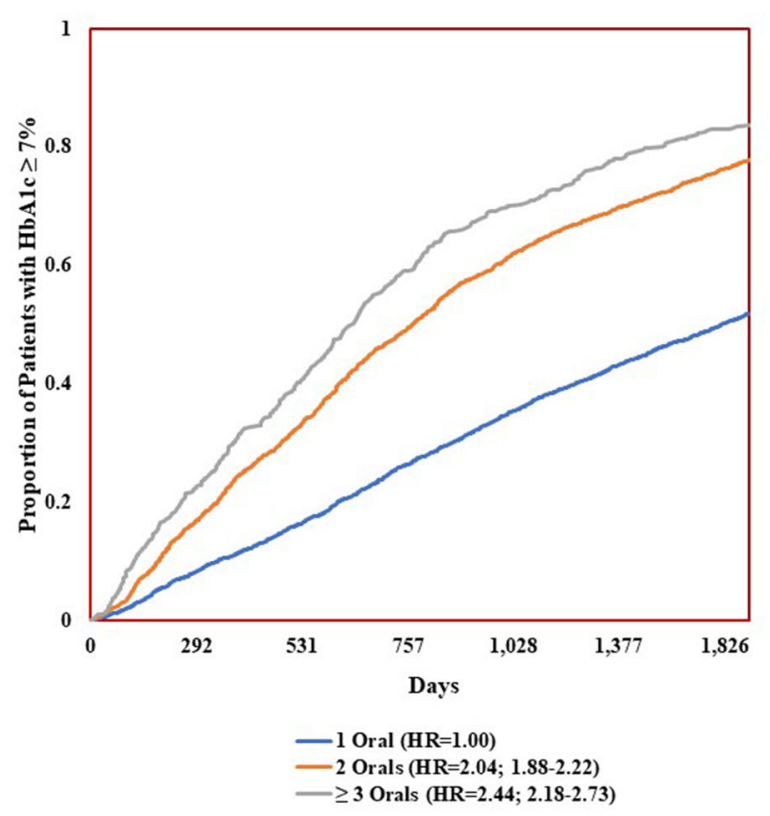

time between $\mathrm{HbAlc}$ tests. Sustained HbAlc $\geq 7 \%$ was defined as having $\geq 2$ consecutive HbAlc laboratory test results above target in the post-period

oral GLAs prescribed at index increased. However, even among those patients who were prescribed an additional therapy, there was often a delay in such a therapy modification. The mean length of time between identification of sustained $\mathrm{HbA} 1 \mathrm{c} \geq 7 \%$ and therapy modification was 401 days (1.1 years). In the multivariable analyses (Fig. 4b), patients treated with two classes of oral GLAs were $49 \%$ (odds ratio [OR] 0.507; 95\% CI 0.423-0.607) less likely to have clinical inertia compared to individuals treated with one GLA while patients treated with three or more classes of oral GLAs were $73 \%$ less likely to add an additional class of GLA (OR 0.268; 95\% CI 0.212-0.339).

As a test of the robustness of the results, all analyses were also conducted using only the subset of patients who had a pre-period HbA1c below target $(N=2392)$. The results of these alternative (sensitivity) analyses were generally consistent with the primary results, as reported above. In both sets of analyses, treatment with 


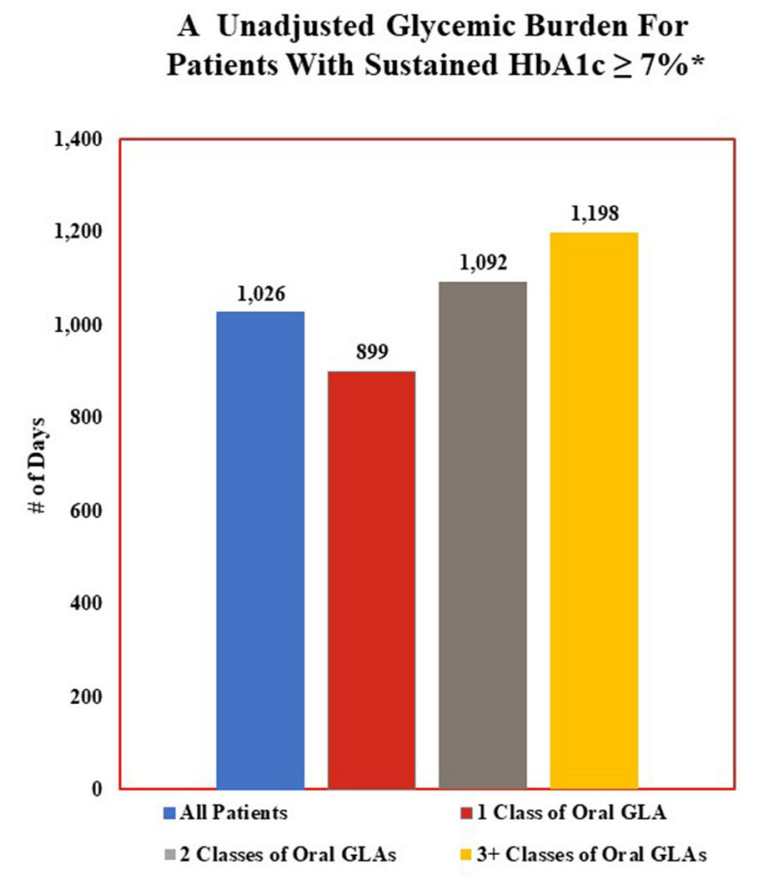

Fig. 3 Glycemic burden and number of classes of oral glucose-lowering agents: descriptive statistics and multivariable Analyses. ${ }^{*}$ Unadjusted differences in results for patients based upon number of classes of oral GLAs prescribed at index date is statistically significant $(P<0.05)$. ${ }^{* *}$ Results from multivariable analyses that controls for age, sex, pre-period general health and length

more classes of GLAs was associated with a statistically significant decrease in the time to sustained HbA1c above target. However, the patients in the subset analysis were generally slower to have sustained HbA1c above target. For example, in the main analyses, $16 \%$ of patients had sustained HbA1c $\geq 7 \%$ after 365 days ( 1 year), while in the sensitivity analyses only five of patients had "failed" on oral GLA therapy after 365 days (1 year). Similarly, for those patients who had sustained HbA1c $\geq 7 \%$, the glycemic burden and likelihood of clinical inertia were also lower in the sensitivity analyses. For example, treatment with two classes of oral GLAs compared to one class, was associated with 1.17-fold the number of days of glycemic burden in the sensitivity analyses (IRR 1.174; 95\% CI 1.073-1.284) compared to 1.22 -fold the number of days in the main analyses. In the sensitivity analyses, treatment
B Incidence Rate Ratios: Association Between Additional Classes of Orals and Number of Days of Glycemic Burden ${ }^{A}$

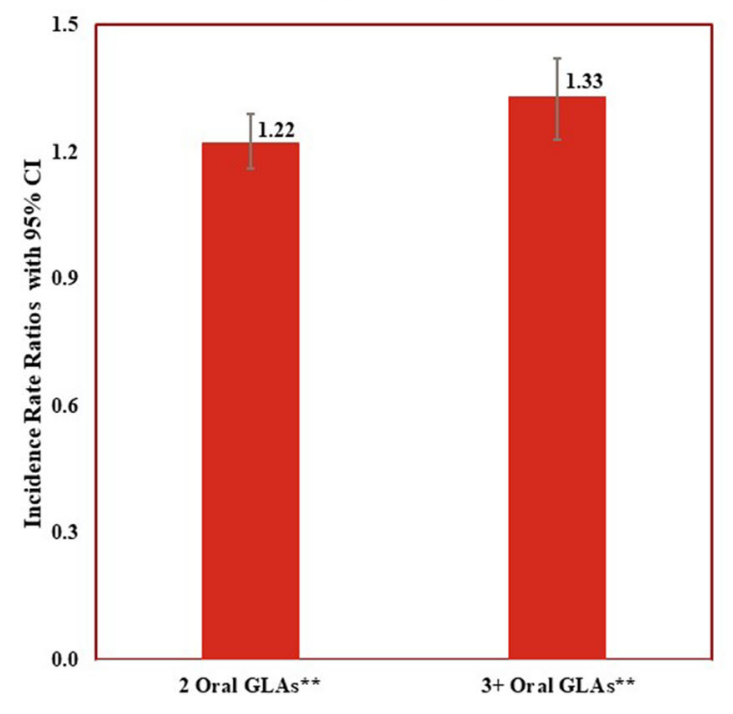

of time between $\mathrm{HbAlc}$ tests indicate significant increase in glycemic burden for patients treated with 2 classes of oral GLAs or $\geq 3$ classes of oral GLAs compared to patients treated with 1 class of oral GLA. Glycemic burden is measured as the percentage of days in the post-period with $\mathrm{HbAlc} \geq 7 \%$ for patients with $\geq 2$ consecutive HbAlc results above target

with two classes of oral GLAs, compared to one class was associated with a $41 \%$ (OR $0.589 ; 95 \%$ CI $0.442-0.785)$ reduction in the likelihood of clinical inertia, while in the main analyses such treatment was associated with a $49 \%$ reduction in the likelihood of clinical inertia. Complete results for the sensitivity analyses are provided in ESM Appendix B.

\section{DISCUSSION}

While clinical inertia in T2D has been a topic of research for many years [10], this study is one of the first to examine the rate of failure on oral GLAs as a medication class, along with subsequent glycemic burden and clinical inertia. As hypothesized, oral monotherapy failed for many of the patients in this US-wide population, and it failed relatively quickly. For 
A Unadjusted Clinical Inertia For Patients With Sustained HbA1c $\geq 7 \%$ *

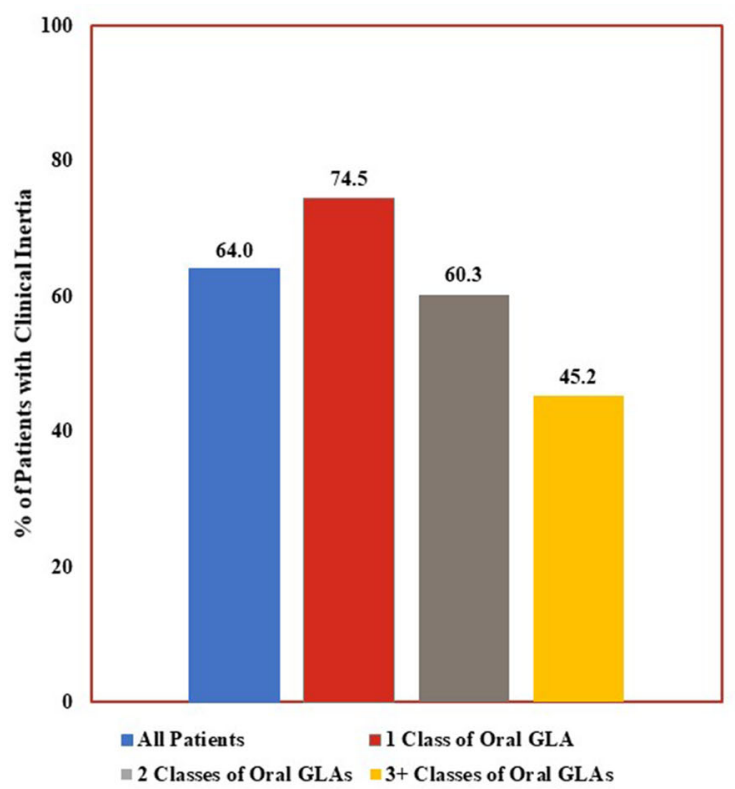

Fig. 4 Clinical inertia and number of classes of oral glucose-lowering agents: descriptive statistics and multivariable analyses. ${ }^{*}$ Unadjusted differences in results for patients based upon number of classes of oral GLAs prescribed at index date is statistically significant $(P<0.05)$. ${ }^{* *}$ Results from multivariable analyses that

instance, after 730 days (2 years), $36 \%$ of patients had sustained HbA1c $\geq 7 \%$ and half of all patients had sustained $\mathrm{HbA} 1 \mathrm{c} 7 \%$ by 1102 days (3.0 years). The glycemic burden associated with such failure was substantial, with affected patients living with above-target hyperglycemia for an average of nearly 3 years (mean 1026 days). Consistent with this large glycemic burden, and also as hypothesized, clinical inertia affected a majority of those unable to sustain $<7 \%$ HbA1c. For instance, at the 5.5 year follow-up, $64 \%$ of patients with sustained $\mathrm{HbA} 1 \mathrm{c} \geq 7 \%$ had never been prescribed an intensified treatment regimen. Meanwhile, those whose therapy was intensified lived for an average of 401 days (1.1 year) with poorly controlled diabetes (i.e., HbA1c $\geq 7 \%$ ) prior to the intensification. The following subsections discuss the main observations in the context of previous research.
B Odds Ratios: Association Between Additional Classes of Orals and Clinical Inertia ${ }^{A}$

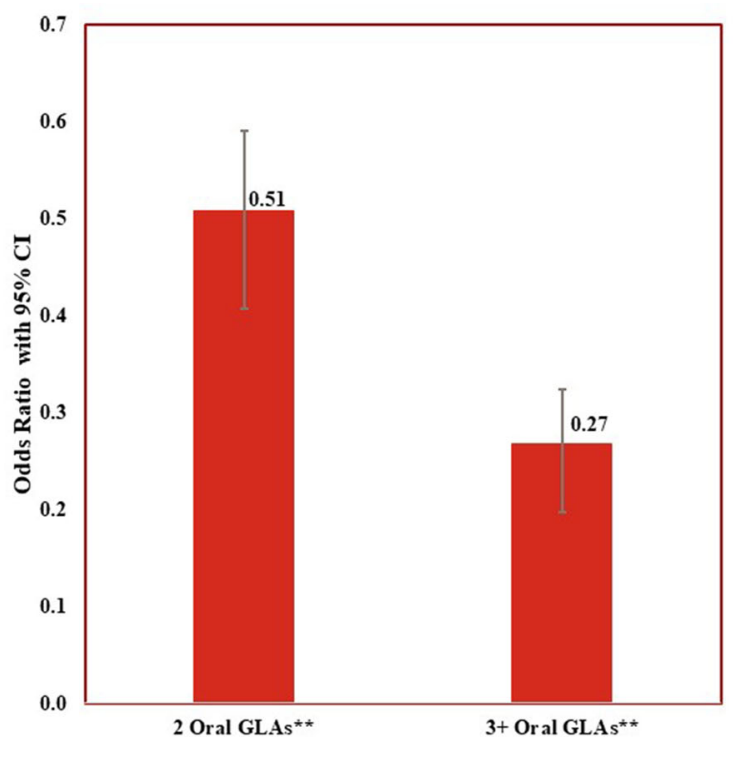

controls for age, sex, pre-period general health and length of time between HbAlc tests indicate significant lower odds of clinical inertia associated with being treated with 2 classes of oral GLAs or $\geq 3$ classes of oral GLAs compared to patients treated with 1 class of oral GLA. Clinical inertia defined as no addition of other classes of GLAs

\section{Time to Treatment Failure on Oral GLAs}

Consistent with the UKPDS finding that hyperglycemia generally increases over time among patients with T2D regardless of their treatment regimen [16], the percentage of patients who failed on oral therapy in this study increased steadily over time and rose to a failure rate of half the population prior to the end of the third year post-initiation. Other clinical trial research has reported lower percentages of failure on oral medications over longer spans of time $[3,17,18]$, likely due to differences in study design (e.g., prospective or retrospective), treatment targets, and even the specific oral drugs examined, given that length of time to treatment failure has been found to vary based on the type of oral therapy used [16]. Meanwhile, a recent study by Romera et al. with a similar design to the current one looked at time 
to failure on oral GLAs and found that many patients were unable to achieve HbA1c $<8 \%$ more than 1 year post-initiation (median days 440 [95\% CI 421-459 days]; mean days 597.7 [standard deviation 459.3]) [13]. In contrast to the present study, however, Romera et al. looked exclusively at individuals with poor glycemic control (HbA1c $\geq 8 \%$ ) and higher treatment complexity (all had obesity and were started on $\geq 2$ oral GLAs). The narrower inclusion criteria likely explain why Romera et al. found that many patients failed on oral therapy from day 1 [13].

Notably, in concert with previous studies [11], the time to failure on antidiabetic therapy decreased in the present study as the number of oral therapies prescribed increased. Similarly, in Romera et al., the length of time to treatment intensification decreased as baseline HbA1c increased, suggesting that oral GLA therapy is less helpful in treating more severe cases of hyperglycemia [13]. As such, these observations support the ADA suggestion that combination therapies be initiated at presentation or soon thereafter for patients with the worst glycemic control (HbA1c $>1.5 \%$ above target) [12].

\section{Glycemic Burden}

The current estimate of glycemic burden, though substantial, is generally consistent with the 0.4 to $>7$ year ranges of time with abovetarget hyperglycemia reported in earlier research [11, 13], although comparisons should be made with caution due to differences in glycemic thresholds and definitions of intensification. Above-target hyperglycemia has been associated with increased risk of microvascular and long-term cardiovascular complications $[6,8,10]$. As such, the large glycemic burden observed in this study represents considerable additional human suffering.

\section{Clinical Inertia}

While clinical inertia has been measured in various ways in studies of T2D [11], the present analyses used two such measures: (1) the median length of time from above-target $\mathrm{HbA1c}$ to treatment intensification, and (2) the proportion of patients on oral therapy with above-target HbA1c who did not receive any treatment intensification during the study period. Looking at time to intensification, among the minority who received modified treatment in this study, descriptive statistics revealed that the roughly 1 -year average time to intensification was generally consistent with the $>1$-year time to intensification reported in most previous research $[10,12]$. The rate of patients who received no intensification during the 5.5 year follow-up period (64\%) was slightly above the $21-63 \%$ range reported in previous investigations with followup periods that exceeded 1 year $[11,13]$. However, this difference is likely due in large part to the more stringent definition of treatment intensification in this study relative to most previous ones. Specifically, in the present study, treatment intensification was defined as the addition of another class of GLA therapy versus an increase in the dosage of the current GLA or prescription of additional GLA. The ADA recommends that treatment intensification occur within approximately 3 months of an above-target HbA1c result and acknowledges that combination therapy eventually becomes necessary, as oral monotherapy typically is insufficient after the first several years of treatment [2]. As evidenced by the present results, however, real-world treatment patterns suggest that few providers comply with this recommendation.

\section{Study Limitations}

The study results should be interpreted in the context of the following limitations. First, these analyses used an administrative claims database and included only patients with medical and prescription coverage as well as recorded HbA1c results, limiting generalizability. Second, patients were identified using diagnostic codes rather than formal diagnostic assessments. Third, the analyses focused on the target of HbA1c $<7 \%$. While the ADA has stated that this target is appropriate for most adults [5], the analyses does not allow for individualization of this target. Fourth, the multivariable analyses were unable to control for potential variables 
which may also be associated with outcomes, such as duration of diabetes and patient socioeconomic status. Fifth, sample size limitations precluded any analyses of the association between any specific GLA or class of GLA and outcomes. Finally, the analyses revealed associations but not causation.

\section{CONCLUSIONS}

This study explored treatment failure on oral GLA therapy and subsequent outcomes among US patients with T2D. In particular, the analyses quantified time to failure on oral GLA therapy and the association between the number of classes of oral GLAs prescribed and the time to therapeutic failure. In addition, for individuals who had sustained HbA1c $\geq 7 \%$, the research quantified the relationship between treatment and glycemic burden and between treatment and clinical inertia. The results suggest that a substantial portion of US patients fail to achieve the ADA treatment target of HbA1c of $<7 \%$ after 3 years on oral GLA therapy, and that patients live with above-target hyperglycemia for an average of 1026 days (2.8 years). Moreover, a majority (64\%) of the patients in this study who failed on oral monotherapy were not prescribed an additional class of GLA to their treatment regimen after having a sustained HbA1c $\geq 7 \%$. While previous research has explored clinical inertia in the treatment of $\mathrm{T} 2 \mathrm{D}$, this is one of the first naturalistic, observational studies to examine treatment failure on oral GLAs as a medication class. The findings illustrate the glycemic burden and clinical inertia associated with oral GLA treatment and suggest that physicians may wish to consider earlier initiation of injectable therapies to potentially minimize the burden of T2D when oral therapies fail.

\section{ACKNOWLEDGEMENTS}

Funding. Sponsorship for this study and publication fees, including rapid service fees, were funded by Eli Lilly \& Co.
Editorial Assistance. Editorial assistance in the preparation of this article was provided by Patricia Platt, who was employed as a sub-contractor for HealthMetrics Outcomes Research for this work.

Authorship. All named authors meet the International Committee of Medical Journal Editors (ICMJE) criteria for authorship for this article, take responsibility for the integrity of the work as a whole, and have given their approval for this version to be published.

Authors' Contributions. K Boye had primary responsibility for the data acquisition; $\mathrm{K}$ Boye and J Kiljański had primary responsibility for the conception and design of the research and critical revisions of the manuscript; MJ Lage had primary responsibility for the statistical analysis and drafting of the manuscript.

Disclosures. K Boye and J Kiljański are employees and shareholders of Eli Lilly \& Co. M Lage was compensated for her work on this research by Eli Lilly \& Co.

Compliance with Ethics Guidelines. All study data were fully de-identified and Health Insurance Portability and Accountability Act (HIPAA) compliant. Given the use of retrospective and de-identified data, ethics committee approval was not required. Permission to use the data for this study was granted by license between IBM Watson Health and Eli Lilly and Company.

Data Availability. The data used in this study are not available from the authors due to licensing restrictions. Permission to use the data for this study was granted by license between IBM Watson Health and Eli Lilly and Company.

Open Access. This article is licensed under a Creative Commons Attribution-NonCommercial 4.0 International License, which permits any non-commercial use, sharing, adaptation, distribution and reproduction in any medium or format, as long as you give appropriate credit to the original author(s) and the source, provide a link to the Creative Commons licence, and 
indicate if changes were made. The images or other third party material in this article are included in the article's Creative Commons licence, unless indicated otherwise in a credit line to the material. If material is not included in the article's Creative Commons licence and your intended use is not permitted by statutory regulation or exceeds the permitted use, you will need to obtain permission directly from the copyright holder. To view a copy of this licence, visit http://creativecommons.org/licenses/by$\mathrm{nc} / 4.0 /$.

\section{REFERENCES}

1. Fonseca VA. Defining and characterizing the progression of type 2 diabetes. Diabetes Care. 2009;32: S151-6.

2. American Diabetes Association. 9. Pharmacologic approaches to glycemic treatment: standards of medical care in diabetes-2020. Diabetes Care. 2020;43:S98-110.

3. Matthews DR, Cull CA, Stratton IM, Holman RR, Turner RC. UKPDS 26: Sulphonylurea failure in non-insulin-dependent diabetic patients over six years. UK Prospective Diabetes Study (UKPDS) Group. Diabet Med. 1998;15:297-303.

4. Kahn SE, Haffner SM, Heise MA, et al. Glycemic durability of rosiglitazone, metformin, or glyburide monotherapy. N Engl J Med. 2006;355:2427-43.

5. American Diabetes Association. 6. Glycemic targets: standards of medical care in diabetes-2020. Diabetes Care. 2020;43:S66-76.

6. Holman RR, Paul SK, Bethel MA, Matthews DR, Neil HAW. 10-year follow-up of intensive glucose control in type 2 diabetes. N Engl J Med. 2008;359: 1577-89.

7. UK Prospective Diabetes Study Group. Intensive blood-glucose control with sulphonylureas or insulin compared with conventional treatment and risk of complications in patients with type 2 diabetes (UKPDS 33) UK Prospective Diabetes Study (UKPDS) Group. Lancet. 1998;352:837-53.

8. Patel A, MacMahon S, ADVANCE Collaborative Group, et al. Intensive blood glucose control and vascular outcomes in patients with type 2 diabetes. N Engl J Med. 2008;358:2560-72.

9. Zoungas S, Arima H, Gerstein HC, et al. Effects of intensive glucose control on microvascular outcomes in patients with type 2 diabetes: a metaanalysis of individual participant data from randomised controlled trials. Lancet Diabetes Endocrinol. 2017;5:431-7.

10. Andreozzi F, Candido R, Corrao S, et al. Clinical inertia is the enemy of therapeutic success in the management of diabetes and its complications: a narrative literature review. Diabetol Metab Syndr. 2020;12:52.

11. Khunti K, Gomes MB, Pocock S, et al. Therapeutic inertia in the treatment of hyperglycaemia in patients with type 2 diabetes: a systematic review. Diabetes Obes Metab. 2018;20:427-37.

12. Davies M, D'Alessio D, Fradkin J, et al. A consensus report by the American Diabetes Association (ADA) and the European Association for the Study of Diabetes (EASD). Diabetalogia. 2018;2018:2461-98.

13. Romera I, Díaz S, Sicras-Mainar A, et al. Clinical inertia in poorly controlled type 2 diabetes mellitus patients with obesity: an observational retrospective study. Diabetes Ther. 2020;11:437-51.

14. Glasheen WP, Cordier T, Gumpina R, et al. Charlson Comorbidity Index: ICD-9 update and ICD-10 translation. Am Health Drug Benefits. 2019;12: 188-97.

15. Glasheen WP, Renda A, Dong Y. Diabetes complications severity index (DCSI) - update and ICD-10 translation. J Diabetes Complications. 2017;31: 1007-13.

16. Wallace TM, Matthews DR. Coefficient of failure: a methodology for examining longitudinal beta-cell function in Type 2 diabetes. Diabet Med. 2002;19: 465-9.

17. Wright A, Burden ACF, Paisey RB, Cull CA, Holman RR. Sulfonylurea inadequacy: efficacy of addition of insulin over 6 years in patients with type 2 diabetes in the U.K. Prospective Diabetes Study (UKPDS 57). Diabetes Care. 2002;25:330-6.

18. Kahn SE, Lachin JM, Zinman B, et al. Effects of rosiglitazone, glyburide, and metformin on $\beta$-cell function and insulin sensitivity in ADOPT. Diabetes. 2011;60:1552-60. 